The National Institute of Health and Welfare, Oulu, Universities of Oulu, Tampere and Helsinki, Finland.

We studied C. trachomatis (CT) serotype-specific antibodies in Finnish women between 1980's and 2000's. C. trachomatis seropositive women $(1,169)$ were available from a subcohort $(11,067)$ of the Finnish Maternity Cohort representing the 1980's $(\mathrm{N}=480)$ and 2000 's ( $\mathrm{N}=331$ ) for serotype specific classification by microimmunofluorescence test. Serotype distributions were comparable in the 1980's and 2000's, G, E and J being the most prevalent. Serotype D peaked in the 1990's. The overall serotype profile changed first between 1980's and 1990's but changed back in the 2000's. The rates of women with antibodies against two or more serotypes increased in the 1990's, but then decreased again. In conclusion, we analysed the rates and distributions of $C T$ serotype-specific antibodies from the 1980's to 2000's in fertile-aged female population in Finland. We found that CT serotype distributions varied considerably over time.

\section{P2.141 KNOWLEDGE, ATTITUDES AND PRACTICES OF HEALTHCARE WORKERS FROM UNIVERSITY HOSPITAL OF OUAGADOUGOU (BURKINA FASO) FACE TO ACCIDENTAL BLOOD EXPOSURE (ABE) AND BIOLOGICAL FLUIDS IN CAREGIVING FACILITIES}

doi:10.1136/sextrans-2013-051184.0405

'Y Sagna, ${ }^{2} \mathrm{~S}$ Kouanda, ${ }^{1} \mathrm{H}$ Tiéno, ${ }^{1} \mathrm{R}$ A K Nianogo, ${ }^{1} \mathrm{R}$ Bognounou, ${ }^{1} \mathrm{~J}$ Y Drabo. 'Internal Medicine Department of Yalgado Ouédraogo University Hospital (CHU YO), Ouagadougou, Burkina Faso; 'Institut de recherche en sciences de la santé, Ouagadougou, Burkina Faso

Objective Accidental blood exposure $(\mathrm{ABE})$ is defined as "any accidental exposure to blood or to a biological fluid contaminated by blood, including a cutaneous injury during an incision or injection, or a projection on mucosa or on an injured skin". we aimed to evaluate the knowledge, attitudes and practises of healthcare workers in one of West Africa country national referral hospital (Yalgado Ouédraogo University Hospital) face to ABE.

Methods A descriptive cross-sectional and analytical study was conducted, from May to July 2009, on all healthcare workers (medical, paramedical and support staff) working for at least a year in this hospital university. A self and anonymous questionnaire was used to gather the information. The knowledge assessment was made using a digital scale. Results 462 healthcare workers responded to the questionnaire (response rate: $51.3 \%$ ). They composed of 60 physicians $(12.9 \%)$, 313 paramedics $(67.7 \%)$ and 89 support officers $(19.2 \%)$. The exact definition of an $\mathrm{ABE}$ was known from 255 healthcare workers $(55.2 \%)$ and $375(81.2 \%)$ were aware of the principles of universal precautions. Medical staff better defined the ABE than the other healthcare workers $(p=0.00) .133$ healthcare workers $(29.4 \%)$ were at least once a victim of an ABE, of which 84 (61.3\%) had not reported their $\mathrm{ABE}$. Ignorance of the support procedures was in $26.8 \%$ of cases the main cause of no report. The risk of contamination after $\mathrm{ABE}$ had not been assessed in $60.3 \%$ of cases. Only $69.7 \%$ of the healthcare workers were aware that HIV is a potentially transmissible infectious agent during an $\mathrm{ABE}$. Protective equipment was not always available and not constantly used.

Conclusion The ABE knowledge of these referral hospital healthcare workers is not satisfactory. Appropriate communication and awareness means on the risks associated with the ABE should be further developed in healthcare settings.

\section{P2.142 T00 BAD! SINGLE MOLECULE ANTIRETROVIRAL DRUGS MAY NOT BE THE MAGIC BULLET}

doi:10.1136/sextrans-2013-051184.0406

'T Agyarko-Poku, ${ }^{2} Y$ Adu Sarkodie. 'Suntreso Government Hospital, Ghana Health Services, Kumasi, Ghana; ${ }^{2}$ School of Medical Sciences, Kwame Nkrumah University of Science and Technology, Kumasi, Ghana
Background Adherence to Antiretroviral drugs (ARVs) poses a challenge for an effective Antiretroviral Therapy in Ghana. The introduction of single molecule ARVs regime was seen as an antidote to non-adherence among HIV patients on treatment. This study was conducted among ARVs experienced patients, who were about to be switched onto single molecules, to determine their perception on the new treatment regime.

Methods 1681 HIV positive patients from Suntreso STI/HIV Clinic in Kumasi, who have been on treatment for more than 12 months and consented to participate in this cross sectional study, were interviewed using structured pretexted questionnaire prior to the commencement of the new single molecule ARVs treatment regime. Data was entered and analysed using SPSS version 16.

Results Whereas $60.9 \%(1023 / 1681)$ find the present dose regime cumbersome, $39.1 \%(657 / 1681)$ prefer the multiple dose regime. $42.2 \%$ (709) of respondents have some reservations about single dose regime whilst $24.6 \%$ (413) prefer it with $33.3 \%(559)$ being indifferent. Reasons for the reservation included; 'Side effect may be too serious' (48.3\%, 342/709), 'Virus too powerful for a single molecule' $(30.2 \%, 214 / 709)$, 'Attempts to deprive us of drugs and facilitate our death' $(13.8 \%, 92 / 709)$ and 'Cost of drugs will be expensive in future for a combine drugs' $(7.7 \%, 55 / 709)$.

Conclusion Although majority of patients find the multiple dose regimes cumbersome, they are sceptical about the use of the single molecule regime. The new regime may result in overdosing if they find it inadequate to provide the needed protection. The fear of serious adverse reaction from combination of ARVs compared with separate drugs, may scare them from taking the treatment. Intensive adherence counselling taking care of the above concerns is essential before patients are switch onto the single molecule ARV regime

\section{P2.143 BENEFITS, CHALLENGES AND LESSONS LEARNED IN IMPLEMENTING AN HIV MEDICATION DELIVERY SCHEME}

doi:10.1136/sextrans-2013-051184.0407

L Hilton, K A Fernando, C Penn, H Jaleel, J H Day. Department of Sexual Health \& HIV Medicine, Southend University Hospital NHS Foundation Trust, Essex, UK

Introduction The HIV department of Southend University Hospital NHS Foundation Trust UK, care for 370 patients of which $80 \%$ are taking antiretroviral therapy(ART).

Monthly ART spend is approximately $£ 200,000$. A proposal for ART home delivery was made by the HIV multidisciplinary team(MDT) and local procurement pharmacists. The desired outcome being patient convenience, whilst making financial savings for the Trust in view of $0 \%$ value added tax for delivered medication.

Methods A delivery scheme began in June 2011. 60\% of patients on ART chose to enter it. 3 months later, however, failed deliveries were reported. This continued despite efforts to rectify issues with the delivery provider.

In November 2011, an alternative provider, with greater experience in ART, was recruited. Now, 15 months later, the scheme continues successfully, with $55 \%$ of patients on ART entered.

Outcomes: Initial patient survey identified potential concerns regarding entering the scheme - firstly, reliability of timing of medication delivery and secondly, protection of patient identifiable information.

The convenience of not waiting for ART to be dispensed in the hospital pharmacy is a key patient reported benefit. From the hospital perspective, home delivery is resulting in savings of $£ 9300$ per month. Additionally, there is less pharmacy storage space consumption.

Additional administration time consumption, however, is noted in 'trouble-shooting' and coordinating the process. 
Conclusion We propose that with good interagency and MDT working, an efficient and effective ART delivery service is feasible, with patient and hospital benefits. Collaborative approaches between the delivery service and HIV MDT should be established and regularly evaluated with patient input. Identifying a key contact person for the delivery service is important to ensure continuity of communication, together with a local contractual service level agreement to ensure clear terms of reference and accountability. Results of a qualitative patient survey evaluating the delivery service are imminent.

\section{P2.144 DO PATIENTS HAVE CONFIDENCE IN TRAINED NON MEDICAL HEALTH CARE STAFF SCREENING FOR SEXUALLY TRANSMITTED INFECTIONS?}

doi:10.1136/sextrans-2013-051184.0408

${ }^{1} \mathrm{R}$ M Jackson, ${ }^{2} \mathrm{M}$ Gill, 'E Foley, 'R Patel. 'Royal South Hants Hospital, Southampton, UK; '2University of Southampton, Southampton, UK

Background In 2011, 1.3 million sexual health screens (SHS) were conducted in genitourinary medicine clinics across the UK, a doubling of workload in the last 8 years. One approach UK clinicians have adopted in managing this increase is for minimally trained non-medical staff (health care support workers (HCSWs)) to deliver protocol driven asymptomatic screening to low risk patients. There is limited research regarding patient confidence with the service offered by HCSWs and re-attendance rates could be an indicator of patient dissatisfaction.

Aim To assess whether patients who have asymptomatic screening with HCSWs were more likely to return for subsequent assessment by a clinician following discharge.

Method A case controlled study of 300 asymptomatic patients attending for sexual health screening between October 2011 and April 2012. There were 2 arms with equal patient numbers, the HCSW led clinic and the clinician led clinic. Data collection and analysis for both groups included patient demographics, diagnoses, treatment, test results and time to next new diagnosis.

Results No significant difference was found between the patient demographics of the two groups. The rates of Chlamydia infection between the HCSW and the clinician groups were $8 \%$ and $7.3 \%$ respectively. Within the clinician group 4 cases of syphilis, 1 new diagnosis of HIV and 1 case of Hepatitis $\mathrm{C}$ were also diagnosed. The HCSW clinic had 19 (12.7\%) patients re-attend for further screening within 11 months compared to $16(10.7 \%)$ patients who saw a clinician, showing no significant difference between the two groups $[p=0.124$ Fishers exact test $]$. Only 1 patient from each group reattended within 6 weeks for further screening due to the development of symptoms.

Conclusion This study highlights low patient re-attendance rates within the HCSW group. This is an indirect marker of patient satisfaction and demonstrates patients are reassured with the service they offer.

\section{P2.145 ADHERENCE TO REPEATED HOME SCREENING FOR BACTERIAL VAGINOSIS AND STIS AMONG YOUNG WOMEN PARTICIPATING IN THE BRAVO RANDOMIZED CLINICAL TRIAL}

doi:10.1136/sextrans-2013-051184.0409

${ }^{1}$ R L Cook, ${ }^{1} \mathrm{C}$ Okafor, ${ }^{2} \mathrm{~S}$ Hillier, ${ }^{2} \mathrm{H}$ Wiesenfeld, ${ }^{3} \mathrm{~S}$ Phillip, ${ }^{4} \mathrm{~A}$ Sena, ${ }^{5} \mathrm{~S}$ Lensing, ${ }^{5} \mathrm{~J}$ Lee, ${ }^{6} \mathrm{~L}$ Saylor, ${ }^{7} \mathrm{~J}$ Schwebke. 'University of Florida, Gainesville, FL, United States; ${ }^{2}$ University of Pittsburgh, Pittsburgh, PA, United States; ${ }^{3}$ San Francisco Department of Public Health, San Francisco, CA, United States; "University of North Carolina, Chapel Hill, NC, United States; ${ }^{5}$ University of Arkansas for Medical Sciences, Little Rock, AR, United States; ${ }^{6}$ FHI 360, Durham, NC, United States; ' University of Alabama, Birmingham, AL, United States
Background The BRAVO trial is an ongoing study designed to determine whether bi-monthly home-screening and treatment for asymptomatic bacterial vaginosis (BV) reduces risk of urogenital chlamydia and gonorrhoea infections in young women. Return rate of self-collected swabs is a critical element of home testing interventions. We conducted this preliminary analysis to determine the swab return rate and to assess its association with age, race, or recruitment setting.

Methods Participants, recruited from 10 clinics in 5 cities, were asked to mail self-collected vaginal swabs to the research team every 2 months for one year. For each evaluable participant, we determined the number of kits returned (of 6 total) and compared the proportion of women that returned all 6 kits according to age, race, and recruitment setting.

Results Data were available for 756 participants who were primarily non-Hispanic Blacks (76\%), with median age 21 (range: 17-25 years), and mostly recruited from STD clinics (82\%). Nearly all women (89\%) returned at least one swab; 59\% returned all 6 kits; $14 \%$ returned 5, $16 \%$ returned $1-4$, and $11 \%$ returned none. Complete swab return rate (all 6) was greater among women aged 23-25 than those aged 17-22 ( $67 \%$ vs. $56 \%, \mathrm{p}<0.01)$ and varied among the 5 recruitment cities (range $42 \%-66 \%, p=0.02$ ). Return rates were not significantly associated with race and Hispanic ethnicity, or with recruitment from STD clinics vs. other settings ( $58 \%$ vs. $66 \%, \mathrm{p}=0.10$ ).

Conclusions The majority of study participants collected and submitted all six home-screening kits, and 73\% completed at least five. Adolescent women aged 22 and younger had a lower return rate than women aged 23-25, although the complete return rate was still over $50 \%$. Therefore, frequent home-screening for BV and STIs is feasible in clinical trial settings and could likely be implemented as part of clinical care and STD prevention programmes.

\section{P2.146 CD4 LYMPHOCYTES COUNT AT FIRST PRESENTATION OF HIV POSITIVE PATIENTS ACCESSING ANTIRETROVIRAL THERAPY AT A DISTRICT HOSPITAL IN GHANA}

doi:10.1136/sextrans-2013-051184.0410

${ }^{1}$ T Agyarko-Poku, 'S Bawa, 'F Adomako-Boateng, ${ }^{1} \mathrm{H}$ Afriyie Siaw, ${ }^{2} \mathrm{Y}$ Adu Sarkodie. ${ }^{1}$ Suntreso Government Hospital, Ghana Health Services, Kumasi, Ghana; ${ }^{2}$ School of Medical Sciences, Kwame Nkrumah University of Science and Technology, Kumasi, Ghana

Background Recent high level of mortality among patients both on clinical care and on Highly Active Antiretroviral Therapy (HAART) has been blamed on late reporting of patients to Hospitals. The study determined the CD4 lymphocytes count levels of HIV positive patient at first presentation at STI/HIV Clinic at Suntreso Government Hospital in Kumasi, Ghana.

Methods This retrospective study reviewed clinical records of 883 positive patients, who reported for treatment at the Suntreso Hospital for the first time within January 2010 to December 2011. The date of first reporting and the level of first CD4 counts were recorded. Socio demographic information was also recorded. Data was analysed using SPSS 16.

Results More than half $(54.9 \%, 485 / 883)$ of all HIV positive patients presented with CD4 count of less than $250 \mathrm{cells} / \mathrm{mm}^{3}$. $20.7 \%(183 / 883)$ reported with CD4 count less than $50 \mathrm{cell} / \mathrm{mm}^{3}$, $9.5 \%$ (84/883) with CD4 count of less than 100 cells $/ \mathrm{mm}^{3}, 24.7 \%$ (218/883) with CD4 count of less than 250 cells $/ \mathrm{mm}^{3}, 16.0 \%$ (141/883) with CD4 count of less than 350 cells $/ \mathrm{mm}^{3}, 10.3 \%$ $(91 / 883)$ with CD4 count of less than 500. Less than a quarter $(18.8 \%, 116 / 883)$ of patients came with CD4 count of 500 cells $/ \mathrm{mm}^{3}$ or more. $70.9 \%$ came with CD4 count of less than 350 cells $/ \mathrm{mm}^{3}$.

Conclusion Patients with HIV infection present late at the HIV clinic at Suntreso Government Hospital. Further study is needed to determine the causes for late presenting at the HIV clinic and address them, since this may account for high mortality among HIV positive patients accessing care. 